Short Communications

\title{
DIAGNOSTIC EFFICACY OF MODIFIED ALVARADO SCORE OVER GRADDED COM PRESSION ULTRASONOGRAPHY
}

\author{
Caren Dsouza ${ }^{1}$, John M artis $^{2} \&$ Vinay Vaidyanathan ${ }^{3}$ \\ ${ }^{1}$ Assistant Professor, ${ }^{2}$ Professor, Department of General Surgery, K.S. Hegde M edical Academy, Nitte University, \\ Deralakatte, M angalore - 575 018, India, ${ }^{3}$ Assistant Professor, Department of E.N.T., Father M uller Medical College, \\ Father Muller Road, Kankanady, Mangalore - 575 002, India. \\ Correspondence \\ Caren D'Souza \\ Assistant Professor, Department of General Surgery, K.S. Hegde Medical Academy, Deralakatte, Mangalore - 575018 \\ Mobile : +91 8147003238 E-mail : drcaren11@gmail.com
}

\begin{abstract}
:
Background:

Acute appendicitis is one of the commonest surgical emergencies. Despite a life time cumulative risk of nearly $7 \%$ its diagnosis remains a challenge. The risks of two primary outcomes must be balanced in the management of presumed appendicitis: perforation and misdiagnosis . The rate of misdiagnosis in certain populations is as high as $40 \%$. Diagnostic aids like modified Alvarado score and ultrasonography can dramatically reduce the negative appendicectomies.
\end{abstract}

\section{Methods:}

Data was collected from 60 patients with complains of right iliac fossa pain over a period of 9 months to our hospital. All patients were categorised using the Alvarado score and Graded compression ultrasonography was done. The preoperative and histological findings were compared with the preoperative diagnosis. The collected data was analysed with regards to various parameters like sensitivity, specificity, predictive values and diagnostic accuracy.

\section{Results:}

In our study of 60 patients, 56 patients underwent appendicectomy out of which a histological confirmation of appendicitis was obtained for 50 patients, giving a negative appendicectomy rate of $12 \%$.

By taking a cut-off point of 7 for the M odified Alvarado score, a sensitivity of $97.56 \%$, specificity of $66.67 \%$, positive predictive value (PPV) of $95.23 \%$, negative predictive value (NPV) of $80 \%$ and accuracy of $87.2 \%$ were calculated. Using the cut-off point of 6 , a sensitivity of $90 \%$ specificity of $50 \%$, PPV of $69.23 \%$, NPV of $80 \%$ and accuracy of $55.56 \%$ were obtained. The sensitivity, specificity, PPV, NPV and accuracy rate of ultrasonography was $92.15 \%, 88.9 \%, 97.19 \%, 66.7 \%$ and $85 \%$, respectively.

\section{Conclusion:}

The presence of a modified Alvarado score $>7$ was found to be a dependable aid in the preoperative diagnosis of acute appendicitis. In cases where the score was negative or equivocal, ultrasonography greatly helped in the diagnosis thereby reducing the incidence of negative appendicectomies.

Keywords: Ultrasonography, Acute appendicitis, modified Alvarado score

\section{Introduction :}

Acute appendicitis is the most common cause of an acute abdomen requiring surgery,

Access this article online Quick Response Code

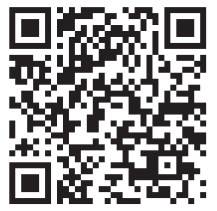

with a lifetime risk of about $7 \%{ }^{1}$. Symptoms of appendicitis overlap with a number of other conditions making its diagnosis a challenge, particularly at an early stage of presentation.
Simple appendicitis can progress to perforation, which is associated with a much higher morbidity and mortality, and surgeons therefore are inclined to operate when the diagnosis is probable rather than wait until it is confirmed ${ }^{2}$.A clinical decision to operate leads to the removal of a normal appendix in $15 \%$ to $30 \%$ of cases. Some cases of appendicitis may resolve spontaneously. None the less, if a period of observation culminates in the diagnosis of a ruptured appendix, the patient may have suffered a poor outcome that was avoidable. Reductions in the 
number of "unnecessary" or non-therapeutic operations should not be achieved at the expense of an increase in number of perforations. ${ }^{2}$

It has been claimed that diagnostic aids can dramatically reduce the number of appendicectomies in patients without appendicitis, the number of perforations, and the time spent in hospital. The various methods advocated to assist in the diagnosis of appendicitis include laparoscopy, scoring systems ${ }^{1}$, , ultrasonography,computed tomography,and magnetic resonance imaging.Imaging techniques have been shown to be particularly accurate. ${ }^{1,2}$

Clinical prediction rules (CPRs) quantify the diagnosis of a target disorder based on findings of key symptoms, signs and available diagnostic tests, thus having an independent diagnostic or prognostic value. CPRs have the potential to reduce diagnostic error, increase quality and enhance appropriate patient care ${ }^{1}$. In 1986, Alvarado constructed a 10-point clinical scoring system, also known by the acronym MANTRELS, for the diagnosis of acute appendicitis as based on symptoms, signs and diagnostic tests in patients presenting with suspected acute appendicitis. However in 1994 Kalan omitted this parameter and produced a modified score. (Table 1)

Graded compression ultrasonography is the least expensive and least invasive of the diagnostic aids and has been reported to have an accuracy of $71 \%$ to $95 \%$. It has been argued that findings at sonography should not supercede clinical judgment in patients with a high probability of appendicitis ${ }^{2}$

\section{Meterials and Methods:}

It was a prospective randomised study conducted at department of general surgery over a period of 9 months. $A$ total of 60 patients participated in the study .Ethics committee approval was obtained for this trial.

INCLUSION AND EXCLUSION CRITERIA: Patients were considered for inclusion in the study if they were referred to the surgical service at Fr mullers medical college mangalore with a provisional diagnosis of acute appendicitis. Patients were excluded from randomisation if they fulfilled any of the following criteria: age less than 14 years; evidence of generalised peritonitis; palpable mass in the right iliac fossa; evidence of acute confusional state or dementia; graded compression ultrasonography already performed.

SCORE: Modified Alvarado score was calculated for all patients and they were divided into 3 groups based on the score group 1: $>7$, group 2:5-6, group $3:<4$. The criteria for diagnosis of appendicitis by modified Alvarado score was a total score of $>7$. The patients with score 5-6 were considered equivocal and score $<4$ were considered negative for appendicitis.

ULTRASONOGRAPHY: Graded compression ultrasonography was done on all patients using Philips HD 11 , high frequency transducer (8-10 hz) . The results were designated positive or negative, by the attending sonographer by using the following criteria: positive-concentrically layered, non compressible sausage like structure in fixed position at the site of maximum probe tenderness, with a diameter $\$ 6 \mathrm{~mm}$.

HISTOPATHOLOGY: 56 patients underwent an appendicectomy. The diagnosis of appendicitis was made on histological grounds on the basis of infiltration of the muscularis propria by neutrophil granulocytes.

\section{Data analysis:}

The collected data was analysed with regard to various parameters like sensitivity, specificity, predictive values and diagnostic accuracy of modified Alvarado score and ultrasonography.

\section{Results:}

A total of 60 patients clinically suspected of having acute appendicitis were included in the study of which 34 were males and 26 females. The incidence of acute appendicitis was highest in the age group 21-30 yrs. Of the 60 patients included in the study, 56 underwent appendicectomy and 4 were conservatively treated. Among those who were operated, 50 had acute appendicitis and 6 were negative. Total of 10 patients had an alternative diagnosis. [Table 2] 
By taking a cut-off point of 7 for the Modified Alvarado score, a sensitivity of $97.56 \%$, specificity of $66.67 \%$, PPV of $95.23 \%$, NPV of $80 \%$ and accuracy of $87.2 \%$ was calculated Using the cut-off point of 6 , a sensitivity of $90 \%$ specificity of $50 \%$, PPV of $69.23 \%$, NPV of $80 \%$ and accuracy of $55.56 \%$ were obtained .The sensitivity, specificity, PPV, NPV and accuracy rate of ultrasonography was $92.15 \%, 88.9 \%$, $97.19 \%, 66.7 \%$ and $85 \%$, respectively .We had a negative appendicectomy rate of $12 \%$.

\section{Criticism of our study :}

We have taken all cases treated conservatively as true negatives. Adequate follow up of these patients was not possible due to which there is always a possibility of a spontaneously resolving appendicitis being missed.

\section{Discussion:}

Acute appendicitis is one of the most common surgical emergencies encountered by junior surgeons' on-call, with emergency appendicectomy making up one in ten of all emergency abdominal surgeries ${ }^{3}$.Over the last hundred years the morbidity and mortality rates related to acute appendicitis have been markedly reduced. This is because of the recognition of the deleterious effects of appendiceal perforation. Thus an aggressive surgical treatment stratergy involving early operation with acceptance of a negative appendicectomy rate of $15-30 \%$ has become universal. It is therefore rightly said that the number of negative appendicectomies is inversely proportional to the number of perforations. ${ }^{4}$

Acute appendicitis may occur at any age, although it is relatively rare at the extremes of age. Previous studies showed that the maximum incidence of the disease occurs in the second decade ${ }^{5}$. Our study a peak incidence was seen between 21-30 yrs.

Several scoring systems have been developed in the diagnosis of acute appendicitis ${ }^{3}$. The Alvarado score, which was developed in 1986, is a simple additive scoring system to help with the diagnosis of acute appendicitis. The classic Alvarado score included left shift of neutrophil maturation (score 1) yielding a total score of 10. However in 1994 Kalan omitted this parameter and produced a modified score . (Table 1)

Using ultrasonography it is possible to confirm appendicitis by visualization of the inflamed appendix or by excluding appendicitis, either by visualisation of normal appendix or by demonstrating an alternative diagnosis like pelvic inflamatory disease, ovarian cyst, right ureteric calculi etc ${ }^{6}$.

When comparing ultrasound to Alvarado score for the diagnosis of acute appendicitis, neither one is significantly advantageous. However false positive rate is reduced to zero when both studies are positive and ultrasound improved the diagnostic efficacy when Alvarado score was negative or equivocal. ${ }^{7}$

Our study showed that patients with Modified Alvarado score $>7$ were more likely to have appendicitis (accuracy $87.2 \%$. The score 5-6 was equivocal with an accuracy of $55.56 \%$. These scores were comparable with the previous studies ${ }^{2}$ [table 3]. The sensitivity and specificity of ultrasonogarphy in our study was comparable to the previousstudies [table 4]

On every surgical take, the investigation of pain in the right iliac fossa differs in men and women. To illustrate this difference, Devey and co-workers ${ }^{8}$ performed a retrospective analysis of 59 cases of pain in the right iliac fossa. They showed that ultrasonography was helpful in excluding alternative diagnosis before appendicectomy. Our study had 10 patients with right iliac fossa pain with alternative diagnosis (table 2)

Out of the 56 patients who underwent appendicectomy, histopathology proved 50 patients to have feature suggestive of appendicitis. This gives us a negative appendicectomy rate of $12 \%$ in our study. This rate is comparable to the previous studies done by AI Quhtani and M ohammad ${ }^{9}$ who had a negative appendicectomy rate of $12.5 \%$ and that of Khan and Rehman ${ }^{10}$ with negative appendicectomy rate of $15.6 \%$.

\section{Conclusion:}

When ultrasound is compared to the modified Alvarado 
score for the diagnosis of acute appendicitis, neither one is significantly advantageous. However, the false positive rate is reduced when both studies are positive. Ultrasound improved the diagnostic accuracy when the Alvarado score was negative or equivocal. Ultrasound is unnecessary when one's degree of clinical suspicion is high. However, the additional information provided by ultrasound does improve diagnostic accuracy in the case of a negative or equivocal Alvarado score.

Polycystic ovary syndrome (PCOS) is a common endocrine disorder affecting about $6 \%$ of women of reproductive age, characterized by gynaecologic and endocrine symptoms including chronic

Table 1: Modified Alvarado Score

\begin{tabular}{|l|c|}
\hline Symptoms & Score \\
\hline M igratory RIF pain & 1 \\
\hline Nausea / Vomiting & 1 \\
\hline Anorexia & 1 \\
\hline Tenderness RIF & 2 \\
\hline Rebound tenderness RIF & 1 \\
\hline Elevated Temperature & 1 \\
\hline Leucocytosis & 2 \\
\hline Total & $\mathbf{9}$ \\
\hline
\end{tabular}

u Score 7-9: Diagnostic of Acute Appendicitis

u Score 5-6: Compatible with Acute Appendicitis but not Convincing

u Score 1-4: Unlikely to Have Acute Appendicitis

Table 2 : final diagnosis

\begin{tabular}{|l|c|}
\hline Diagnosis & Cases \\
\hline Acute appendicitis & 50 \\
\hline PID & 1 \\
\hline Rt ureteric calculi & 3 \\
\hline Intestinal ascariasis & 1 \\
\hline Ovarian cyst & 1 \\
\hline Mittleschmertz & 2 \\
\hline Inconclusive & 2 \\
\hline
\end{tabular}

Table 3 :comparison of Modified Alvarado score with ultrasonography

\begin{tabular}{|l|c|c|c|}
\hline & Score $>7$ & Score 5-6 & ultrasonography \\
\hline sensitivity & $97.56 \%$ & $90.00 \%$ & $92.15 \%$ \\
\hline Specificity & $66.67 \%$ & $50.00 \%$ & $88.90 \%$ \\
\hline PPV & $95.23 \%$ & $69.23 \%$ & $97.19 \%$ \\
\hline NPV & $80.00 \%$ & $80.00 \%$ & $66.70 \%$ \\
\hline Accuracy & $87.20 \%$ & $55.56 \%$ & $85.00 \%$ \\
\hline
\end{tabular}

Table 4 : Comparison of sensitivity and specificity of ultrasonography with other studies

\begin{tabular}{|l|c|c|}
\hline Ultrasonography & Sensitivity & Specificity \\
\hline Our study & $92.15 \%$ & $88.9 \%$ \\
\hline Ramachandra and co workers ${ }^{11}$ & $90.0 \%$ & $96.0 \%$ \\
\hline Charles Douglas and co workers $^{2}$ & $94.7 \%$ & $88.9 \%$ \\
\hline George M atthews and co-workers $^{12}$ & $90.9 \%$ & $88.1 \%$ \\
\hline
\end{tabular}

\section{References :}

1. Ohle R, O'Reilly F, O'Brien K, Fahey T, Dimitrov B .The alvarado score for predicting acute appendicitis: a systematic review .BM C M edicine 2011, 9:139 doi:10.1186/1741-7015-9-139.

2. Douglas C D. Randomised controlled trial of ultrasonography in diagnosis of acute appendicitis, incorporating the alvarado score. BM J 2000; 321:919.

3. Chong CF, Thien A, Mackie A J A, Tin A S, Tripathi S, Ahmad M A, Tan L T, Ang SH, Telisinghe PU. Comparison of ripasa and alvarado scores for the diagnosis of acute appendicitis. Singapore M ed J 2011; 52(5): 340.

4. Rioux M. Sonographic detection of the normal and abnormal appendix. Ajr am roentgenol 1992; 158 (4):773-8.

5. Soltan H M , El-Sherif A M H, M oustafa A HN. The impact of sonography and alvarado score on the clinical management of patients with acute appendicitis. EL-M INIA M ED; VOL 15 (1):2004: 144-157.

6. Puylaert JBCM. Ultrasonography of the acute abdomen: gastrointestinal conditions. Radiol clin n am .2003; ( I41):1227-1242.

7. Stephens PL, Mazzucco J. Comparison of ultrasound and the alvarado score for the diagnosis of acute appendicitis. Conn Med .1999; 63(3): 137-40.

8. Devey L, Henderson J, Wakefeild C. Appendicitis is a separate clinical entity in men and women .BMJ:2001; 323-50.

9. Quatani HH, M uhammad AA. Appraisal of the modified alvarado score for acute appendicitis in adults. jAyub M ed Coll; 2003:24.

10. Khan I, Rehman AU. Application of alvarado scoring system in diagnosis of acute appendicitis.J Ayub M ed Coll; 2005:17(3).

11. Ramachandran P, Sivit CG, Newman KD, Schwartz MZ. Ultrasonography as an adjunct in the diagnosis of acute appendicitis. J padiatr surg. 1996; 31(1):164-7.

12. Sun, Noh, Hyun W, M in, Young G, Lee, Jei, Kim, Park, Kim JH. Receiver operating characteristic analysis of the diagnostic performance of a computed tomographic examination and the Alvarado score for the diagnosing acute appendicitis: emphasis on age and sex of the patients. Journal of computer assisted tomography.2008;32(3) :386391. 